\title{
Acknowledgement to Reviewers of Biomimetics in 2018
}

Biomimetics Editorial Office

MDPI, St. Alban-Anlage 66, 4052 Basel, Switzerland

Published: 17 January 2019

Rigorous peer-review is the corner-stone of high-quality academic publishing. The editorial team greatly appreciates the reviewers who contributed their knowledge and expertise to the journal's editorial process over the past 12 months. In 2018, a total of 32 papers were published in the journal, with a median time to first decision of 25.5 days and a median time to publication of 48 days. The editors would like to express their sincere gratitude to the following reviewers for their cooperation and dedication in 2018:

Adil, Syed Farooq

Alam, Mohammad Parvez

Aljabali, Alaa

Araromi, Oluwaseun

Ardelean, Lavinia Cosmina

Aydin, Yasemin O.

Ball, Vincent

Barth, Friedrich G.

Bax, N.A.M. (Noortje)

Bellardita, Carmelo

Belli, Sema

Benetatos, Panayotis

Berns, Karsten

Bhounsule, Pranav A.

Bibb, Richard

Bortot, Eliana

Boschloo, Gerrit

Carmona-Ribeiro, Ana M.

Chander, Harish

Chen, Lingxin

Cho, Nam-Joon

Cirera, Jordi

Crescenzi, Orlando

Culha, Utku

Dickerson, Andrew

Edwards, Donald H.

Ergin, F. Gökhan

Fitl, Přemysl

Fontanella, Chiara Giulia

Fujiwara, Tomoko

Ghosh, Debanjana

Gianneschi, Nathan

Giorgio-Serchi, Francesco
Giraud, Marie Noëlle

Gómez-Graña, Sergio

Gudwin, Ricardo Ribeiro

Hafuka, Akira

Hammond III, Frank L

Hanafiah, Megat Ahmad Kamal Megat

Hansma, Helen

Harnett, Cindy K.

Hauser, Helmut

Heepe, Lars

Hoffmann, Rolf

Hong, Seonki

Humpolíček, Petr

Ijspeert, Auke

Iop, Laura

Ishikawa, Takuji

Jaudzems, Kristaps

Kaewpirom, Supranee

Kang, Rongjie

Khadiv, Majid

Klosterhalfen, Bernd

Kong, Tiantian

Kono, Hitoshi

Kramer-Bottiglio, Rebecca

Kucinska-Lipka, Justyna

Kumagai, Hiromu

Kunz, Werner

Kyratsis, Panagiotis

Li, Suyi

Liebscher, Jürgen

Liu, Tingyi

Liu, Yahua

Londoño López, Martha Elena 
Lowe, Andrew

\section{Luo, Cheng}

Martinez, Ramses V.

Martins, Luis R. M.

Masouros, Spyros

Mendelson, Leah

Mohades Kasaei, Seyed Mohammadreza

Mohammed, Abdul Samad

Morin, Stephen

Mouchet, Sébastien

Mutyala, Kalyan

Muzykantov, Vladimir

Nagel, Jacquelyn K.

Najarian, Siamak

Nishioka, Yasutaka

Oh, Dongyeop X.

Papavassiliou, Dimitrios

Patil, Madhav

Pereira, Eduardo

Perinelli, Diego Romano

Pezzella, Alessandro

Polygerinos, Panagiotis

Poppinga, Simon

Prilutsky, Boris

Quinn, Roger

Rai, Prakash

Ramasamy, Mohankandhasamy

Rerat, Michel

Rodrigue, Hugo

Rokosz, Krzysztof

Romero, Eder

Sabaté, Manel

Sadati, Hadi

Samanta, Devleena

Schniepp, Hannes
Schuster, Bernhard

Shafti, Ali

Sheng, Jian

Shepherd, Robert F.

Sherman, Benjamin

Sigthorsson, David O.

Song, Young Min

Steward, Robert

Sun, Ziqi

Takaffoli, Mahdi

Tobias, Gerard

Vallet-Regí, María

Van Der Zwaag, Sybrand

Vattikuti, Surya Veerendra Prabhakar

Vazquez Martinez, Juan Manuel

Vincent, Julian F. V.

Voigt, Dagmar

Walker, Ian D.

Wang, Xiaolei

Wang, Liyu

Wei, Qi

Weissburg, Marc

Williams, John A.

Wilts, Bodo

Wright, Cameron H. G.

Wysokowski, Marcin

$\mathrm{Xi}$, Weixian

Young, John

Yudha, Salprima

Zafar, Muhammad

Zelei, Ambrus

Zhang, Tingnan

Zhang, Yingyue

Zhang, Houxiang

Zhu, Quanmin

(C) 2019 by the authors. Licensee MDPI, Basel, Switzerland. This article is an open access article distributed under the terms and conditions of the Creative Commons Attribution (CC BY) license (http://creativecommons.org/licenses/by/4.0/). 